\title{
Liver fat content might be an appropriate measure for estimation of cardiovascular disease risk in non-alcoholic steatohepatitis patients
}

\author{
Raika Jamali ${ }^{1}$, Saeed Pourhassan*2 (1), Nastaran Maghbouli ${ }^{3}$, Haleh Ashraf $^{4}$, Amir Ali Sohrabpour ${ }^{5}$ \\ Received: 25 Oct $2019 \quad$ Published: 12 Oct 2020
}

\begin{abstract}
Background: Non-alcoholic steatohepatitis (NASH) is increasing worldwide due to the metabolic syndrome epidemy. According to the current evidence, a higher cardiovascular disease risk (CVDR) is observed in NASH individuals than the general population. Objective: The relationship between liver fat content (LFC) and CVDR in a cohort of NASH patients was evaluated in this research. Methods: Consecutively selected patients with increased aminotransferase levels and fatty change in liver ultrasonography were enrolled in the study. Those with known causes of viral hepatitis, any hepatotoxic medications or alcohol consumption, autoimmune hepatitis, cigarette smoking, and ischemic heart disease were excluded from the project. The remaining was presumed to have NASH. The Framingham risk score (FRS) and LFC were calculated by means of an online calculator and a valid formula, respectively. The correlation between LFC and independent variables was measured using the Pearson correlation test. The P-value of less than 0.05 was considered significant. The statistical analysis was performed using SPSS program version 18.

Results: Finally, two hundred NASH patients were included in the study. Considering diabetes mellitus as a confounder, there was a fair relationship between LFC and FRS $(\mathrm{R}=0.26$ and 0.23 , respectively, $\mathrm{p}<0.05)$ in the second and third visits. Even after adjustment for known cardiovascular risk factors, LFC was associated with increased CVDR (OR=9.181; 95\% CI: 2.00-42.14, $\mathrm{p}=0.01$ ). The cutoff value of $9.1 \%$ for LFC had a sensitivity of $92 \%$ and a specificity of $87 \%$ for discrimination of the FRS $>20 \%$ and $<20 \%$.

Conclusion: LFC might independently be correlated with CVDR in NASH patients. If further research confirmed this relationship, the inclusion of LFC into the FRS formula would provide an appropriate CVDR estimation tool in NASH.
\end{abstract}

Keywords: Non-alcoholic fatty liver disease, Cardiovascular disease, Fatty liver, Coronary artery disease

Conflicts of Interest: None declared

Funding:This study was financially supported by Tehran University of Medical Sciences (Grant No.1396.3404).

\section{*This work has been published underCC BY-NC-SA 1.0 license.}

Copyright $@$ Iran University of Medical Sciences

Cite this article as:Jamali R, Pourhassan S, Maghbouli N, Ashraf H, Sohrabpour AA. Liver fat content might be an appropriate measure for estimation of cardiovascular disease risk in non-alcoholic steatohepatitis patients.Med J Islam Repub Iran. $2020(12$ Oct);34:135. https://doi.org/10.47176/mjiri.34.135

\section{Introduction}

Non-alcoholic fatty liver disease (NAFLD) includes a range of liver cell damage. It consist of simple liver steatosis, nonalcoholic steatohepatitis (NASH), andcirrhosis (1). The disease burden is growing rapidly due to the epidemic of obesity worldwide (2). Previous leading causes

Corresponding author:Dr Saeed Pourhassan, spourhassan@sina.tums.ac.ir

1. Research Development Center, Sina Hospital, Tehran University of Medical Sciences, Tehran, Iran

2. Department of Internal Medicine, Shariati Hospital, Tehran University of Medical Sciences, Tehran, Iran

3. Department of Physical Medicine and Rehabilitation, Tehran University of Medical Sciences, Tehran, Iran

4. Cardiac Primary Prevention Research Center, Tehran Heart Center, Tehran University of Medical Sciences, Tehran, Iran

5. Digestive Diseases Research Center, Digestive Diseases Research Institute, Shariati hospital, Tehran University of Medical Sciences, Tehran, Iran of end-stage liver diseases including viral hepatitis have been well controlled during the recent decades; meanwhile, NASH is the most common cause of cirrhosis and liver transplantation (3-6). NASH is already considered as the hepatic manifestation of insulin resistance syndrome

$\uparrow$ What is "already known" in this topic:

A higher cardiovascular disease risk (CVDR) is observed in Non-alcoholic steatohepatitis (NASH) individuals than the general population. There is still a paucity of the literature regarding which metabolic index would appropriately estimate the CVDR in NASH patients.

$\rightarrow$ What this article adds:

This experiment evaluated the liver fat content (LFC) as the possible metabolic index to predict the CVDR accurately. During a short-term follow-up time, the observations showed that LFC might independently be correlated with CVDR in NASH patients. 
(7). There is a growing body of evidence suggesting that NASH patients have a higher risk of cardiovascular events in comparison with the normal population. It is worth noting that cardiovascular events are the most causes of mortality and morbidity among NASH patients $(8,9)$.

There is already no clear guideline regarding cardiovascular disease risk (CVDR) assessment in NASH patients $(10-12)$. It seems to be a reasonable approach to modify risk factors and control cardiovascular morbidity and mortality in such patients. Some studies have shown that NASH subjects have higher 10-year CVDR than individuals without NASH $(13,14)$. However, there is still a paucity of literature about the association of liver fat content (LFC) and CVDR in NASH. In line with a previous research, we used "NAFLD liver fat score" in this study to estimate the LFC (15). The aim of the current investigation is to assess the correlation between LFC and CVDR in a cohort of NASH subjects during a six-month follow up period.

\section{MethodsStudy design}

In view of NAFLD mean prevalence in earlier researches in Iran and using this formula: $\mathrm{N}=(\mathrm{t} / \mathrm{d}) 2 *(1-\mathrm{p}) / \mathrm{p}$ $(\mathrm{t}=1.96, \mathrm{p}=0.33$, and $\mathrm{d}=0.2)$, a sample size of 194 subjects was considered in this project (16). In the first phase, consecutively selected patients with increased aminotransferase levels and fatty change in liver ultrasound were enrolled. They were referred to a general hospital gastroenterology clinic from September 2017 to September 2018. At the second phase, individuals with consumption of hepatotoxic medications during the last 6 months, alcohol use or any other known liver diseases, significant systemic comorbidities, cancer, cigarette smoking, and ischemic heart disease were excluded from the study. In the third phase, those with viral hepatitis, autoimmune hepatitis, Wilson disease and hemochromatosis were excluded (17). The remaining participants were presumed as NASH patients. The study measurements, including clinical and laboratory investigations, were performed at baseline, 3 months, and 6 months during the study period.

\section{Evaluation of comorbidities}

The ischemic heart disease risk factors and demographic information were evaluated by a questionnaire. Anthropometric indices and body mass index (BMI) were determined. Diabetes mellitus was defined based on the patient declaration, or if fasting blood glucose $\geq 126 \mathrm{mg} / \mathrm{dl}$ (checked twice), or $\mathrm{HbAlc} \geq 6.5$. Hypertension was defined if the patient notified himself, or the measured blood pressure was higher than 140/90 mmHg (17). Hyperlipidemia was diagnosed if serum fasting triglyceride levels were more than $150 \mathrm{mg} / \mathrm{dl}$, or serum total cholesterol levels were more than $200 \mathrm{mg} / \mathrm{dl}$, or if the patients already used lipid-lowering agents (18). Adult Treatment Panel III (ATPIII) criteria was used for defining metabolic syndrome (19).

\section{Laboratory investigations}

Blood samples were collected following a 12-hour overnight fasting. The laboratory measurements were done by ELISA method using Hitachi autoanalyzer 704 (Roche, Switzerland). The Pars Azmoon reagent Kits (Tehran, Iran) were applied for conducting the assessments. These observations were conducted according to the manufacturers' manual based on previous projects (20, 21). ALT levels of more than $40 \mathrm{U} / \mathrm{L}$ were considered abnormal according to Merck manual (22).

\section{Framingham risk score}

The Framingham risk score (FRS) is a tool for the estimation of 10 -year CVDR $(23,24)$. This score is validated in different worldwide populations, including the Iranian population (25). We used the following web-based calculator to compute FRS in this study: (https://www.qxmd.com/calculate/calculator_252/framing ham-risk-score-2008). Patients with $\mathrm{FRS}^{-}>20 \%$ were considered as high-risk groups according to the American Heart Association (AHA) cardiovascular risk-assessment guidelines (23).

\section{Liver fat content (LFC)}

Based on large cohort studies with more than 10 years of follow-up, "liver fat score" is one of the best prediction scores for estimating cardiovascular and liver-related mortality (13). This score applies the metabolic syndrome and type 2 diabetes mellitus existence, fasting serum insulin and amino transaminases levels. The mentioned LFC formula seems to be an accurate instrument to detect mild to moderate steatosis (26).

\section{Statistical analysis}

The continuous variables were expressed as mean and standard deviation (SD), while the qualitative values are provided as frequencies. The correlation between LFC and independent variables was measured using the Pearson correlation test. Moreover, the relationship between LFC and the 10-year Framingham risk score was calculated by partial correlation considering diabetes mellitus status as a confounder. The LFC changes in different visits were analyzed using ANOVA. Multivariate logistic regression analysis was applied to discriminate which variables were independently related to CVD. Age, gender, waist circumference, HTN, DM and smoking were included in a multivariate linear regression analysis as potential confounders. The Odds ratios (ORs) and their 95\% confidence intervals (CI) were defined for each independent variable.Finally, the LFC cut-off values were calculated for discriminating the "low risk" and "high risk" FRS groups, using receiver operating characteristic (ROC) analysis. SPSS program (version 18) was used for statistical analysis.

\section{Ethical considerations}

Ethics committee of Tehran University of Medical Sciences approved the current research protocol (No.1396.3404). All participants filled an informed written informed consent before enrollment in the project.

\section{Results}

Two hundred patients (50\% male and 50\% female) participated in the study with an age range of 40 to 65 years 
Table 1.Patient charachteristics during the study period

\begin{tabular}{|c|c|c|c|c|c|c|c|}
\hline \multirow[t]{2}{*}{ Variable } & \multicolumn{2}{|c|}{ First Visit } & \multicolumn{2}{|c|}{ Second Visit } & \multicolumn{2}{|c|}{ Third Visit } & \multirow[b]{2}{*}{$\mathrm{p}$} \\
\hline & Mean \pm SD & Min-Max & Mean \pm SD & Min-Max & Mean \pm SD & Min-Max & \\
\hline Body Mass Index $\left(\mathrm{Kg} / \mathrm{m}^{2}\right)$ & $32 \pm 4.9$ & $18.6-48.9$ & $31.7 \pm 4.8$ & $18.3-48$ & $27.8 \pm 5.9$ & $6.4-449$ & $<0.001$ \\
\hline Fasting Blood Sugar (mg/dL) & $106.2 \pm 14.9$ & $75-144$ & $99.5 \pm 10.8$ & $70-126$ & $97.1 \pm 10.9$ & $68-121$ & $<0.001$ \\
\hline Fasting Serum Insulin $(\mathrm{Miu} / \mathrm{L})$ & $20.6 \pm 5.7$ & $10-32$ & $19.4 \pm 5.1$ & $10-29$ & $19.0 \pm 5.2$ & $9-30$ & $<0.001$ \\
\hline Triglyceride $(\mathrm{mg} / \mathrm{dL})$ & $164.3 \pm 93$ & $43-531$ & $130.5 \pm 66.1$ & $40-410$ & $126.8 \pm 60.9$ & $43-400$ & $<0.001$ \\
\hline High Density Lipoprotein (mg/dL) & $34.6 \pm 3.6$ & $27-55$ & $33.5 \pm 3.0$ & $27-49$ & $33.2 \pm 2.9$ & $28-49$ & $<0.001$ \\
\hline Aspartate Aminotransferase(U/L) & $26.7 \pm 13.4$ & $12-99$ & $27.3 \pm 13.2$ & $9-134$ & $29.3 \pm 13.2$ & $9-103$ & 0.001 \\
\hline Alanine Aminotransferase (U/L) & $52.7 \pm 51.7$ & $10-345$ & $44.8 \pm 36.9$ & $9-325$ & $43.8 \pm 32.8$ & $12-248$ & $<0.001$ \\
\hline Alkaline Phosphatase (U/L) & $271.7 \pm 170.6$ & $72-1184$ & $239.4 \pm 126.9$ & $34-796$ & $221.5 \pm 127.0$ & $60-956$ & $<0.001$ \\
\hline Liver Fat Content $(\%)$ & $12.9 \pm 6.5$ & $2.1-43.8$ & $11.9 \pm 5.4$ & $2.5-28.0$ & $11.9 \pm 5.6$ & $2.5-40$ & 0.001 \\
\hline
\end{tabular}

Table 2.Partial correlation analysis between liver fat content and 10-Year Framingham risk score

\begin{tabular}{lccc}
\hline Visit & Gender & $\mathrm{R}$ & $\mathrm{p}$ \\
\hline First & Male & 0.381 & $<0.001$ \\
& Female & 0.072 & 0.472 \\
Second & Male & 0.381 & $<0.001$ \\
& Female & 0.442 & $<0.001$ \\
Third & Male & 0.383 & $<0.001$ \\
& Female & 0.402 & $<0.001$ \\
\hline
\end{tabular}

Table 3.Comparison of liver fat content in patients with Framingham risk score of $>20 \%$ and $<20 \%$ during the study period

\begin{tabular}{lcccc}
\hline & Visit & $\begin{array}{l}\text { FRS } \geq 20 \% \\
(\mathrm{Mean} \pm \mathrm{SD})\end{array}$ & $\begin{array}{l}\text { FRS }<20 \% \\
(\mathrm{Mean} \pm \mathrm{SD})\end{array}$ & $\mathrm{p}$ \\
\hline \multirow{3}{*}{ Liver Fat Content } & First & $14.825 \pm 5.977$ & $6.334 \pm 3.792$ & $<0.001$ \\
& Second & $14.345 \pm 4.478$ & $8.457 \pm 4.891$ & $<0.001$ \\
\hline
\end{tabular}

FRS: Framingham risk score

(mean $\pm \mathrm{SD}: 51.6 \pm 2.7$ ). The summary of clinical features, laboratory data, FRS, and LFC in the study population is shown in Table 1. The metabolic syndrome was found in $80 \%$ of participants. In particular, DM and/or hypertension were present in $75 \%$ of the population. The calculated 10 -year CHD risk $\geq 20 \%$ in patients with NAFLD was $77.5 \%$.

\section{Correlation between LFC and FRS}

Considering diabetes mellitus as a possible confounder and using a partial correlation test, no significant relationship was found between LFC and FRS in the first visit. Meanwhile, there was a fair relationship between the mentioned variables in the subsequent second and third visits (Table 2). The mean LFC was higher in high-risk group patients (FRS $>20 \%$ ) than those with the low-risk group (FRS $<20 \%$ ) in all visits (Table 3 ). In multivariate regression analysis, after adjustment for possible cardiovascular risk factors (DM, HTN, age, gender, waist circumference and smoking), LFC was found to be associated independently with an increased risk of cardiovascular diseases $(\mathrm{OR}=9.183$; 95\% CI: 2.001-42.143, $\mathrm{p}=0.011)$.

\section{The best LFC cut off value for differentiating CVDR groups}

TheLFC optimal cut-off value for discrimination of "low-risk" and "high-risk" FRS groups is illustrated in Figure 1 . The LFC cut-off value of $9.1 \%$ had a sensitivity of $92 \%$ and a specificity of $87 \%$ for the discrimination oflow and high CVDR groups (Fig. 2).

\section{Discussion}

This study evaluated the correlation between LFC and the Framingham 10-year CVDR score in a group of NASH outpatients visited in a referral hospital. The main

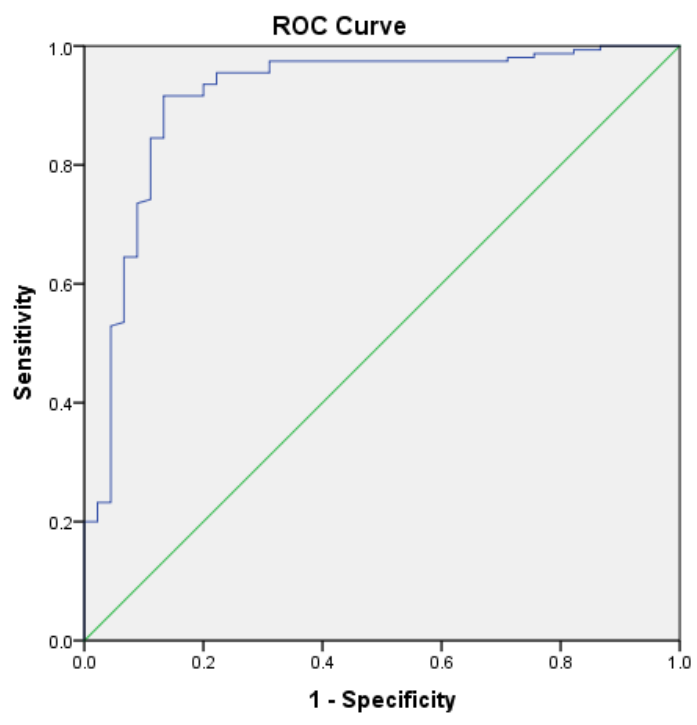

Diagonal segments are produced by ties.

Fig. 1. Receiver operating characteristic (ROC) curve of liver fat content for prediction of high cardiovascular risk group. [Area Under Curve (AUC): 0.91 (0.86-0.97), $\mathrm{p}<0.001$ ]. 


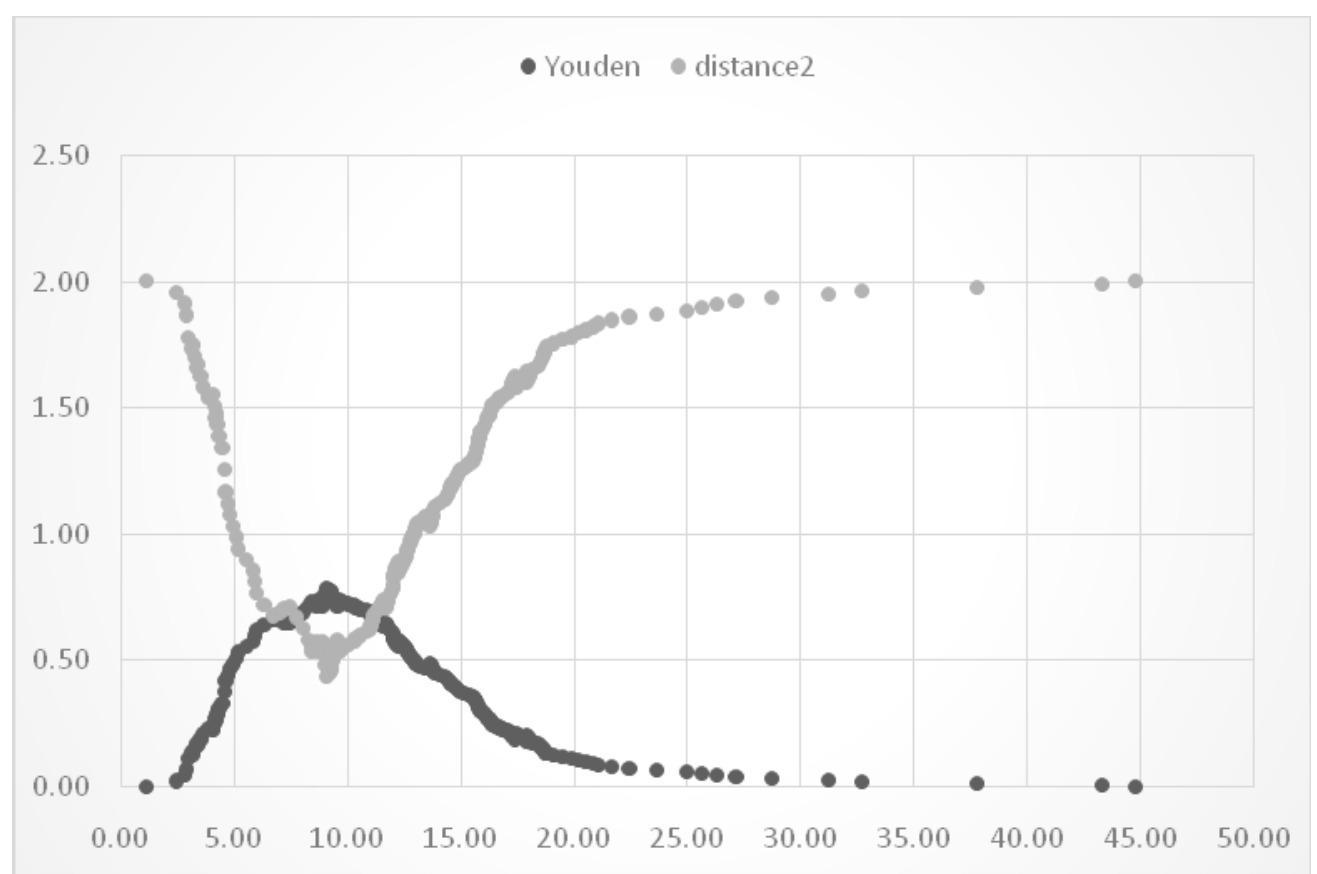

Liver fat content $(\%)$

Fig. 2. The optimal cut point of liver fat content for differentiating high-risk cardiovascular disease from low-risk group ( cutoff $=9.1 \%$ ).

finding in this research was the association between LFC and increased risk of CVD in 6 months' follow up period. Our results are in parallel with the previous studies that suggested NASH as an independent risk factor for coronary artery disease and cardiac-related mortality in Asia and Europe (27-31). Meanwhile, a large prospective study of patients with the acute coronary syndrome concluded that NAFLD independently increased the risk of coronary artery disease (32). Moreover, another investigation demonstrated the association between FRS and NASH fibrosis score (33).

On the other hand, the Diabetes Heart Study suggested that fatty liver is less likely to be a direct mediator of CVD. It proposed that fatty liver is an epiphenomenon in metabolic syndrome (34). At the same time, a populationbased study concluded that fatty liver is not a causal factor for the development of IHD (35).

The controversy in the result of the mentioned investigations might be encountered due to several reasons. First, we should pay attention to the study group characteristics' regarding CVD risk factors. A complete panel of IHD risk factors (including age, low physical activity, smoking, and components of metabolic syndrome) must be addressed in the methodology. The difference in checking these confounding variables describes the discrepancies in the results of correlation analysis. In addition, the method of LFC estimation might also be responsible for the diversity of previous research results.

LFC was independently associated with CVDR in our project after adjusting for the effect of diabetes mellitus as a possible confounding factor. Meanwhile, some explorations recommended that NASH could induce endothelial damage unrelated to CVDR factors $(36,37)$. Additionally, NASH may cause coronary artery calcifications, lead to an increase in carotid intima-media thickness, and generalized arterial stiffness (38). It was anticipated as a subclinical indicator of atherosclerosis, independent of other risk factors for CVD (39).

LFC decreased during the second and third visits in this study. This is probably due to lifestyle change, DM and HTN control. The management of co-morbidities (such as hypertension, dyslipidemia, and lifestyle change) is an effective method for NASH treatment (40).

Finally, the LFC cut-off value for differentiating between "low-risk" and "high-risk" FRS groups was calculated by ROC analysis. The LFC cut-off value of $9.1 \%$ had a sensitivity of $92 \%$ and a specificity of $87 \%$ for discrimination of low and high CVDR groups.

The strength of our study is the utilization of "Liver Fat Score", a highly specific and sensitive tool for LFC calculation. We also considered the FRS for the estimation of 10-year CVDR in our study. This score confirmed to be a strong model ( $\mathrm{C}$ index of 0.83 in women and 0.79 in men) for predicting CVDR in a cohort of Iranian patients (25). This is in line with the previous exploration that emphasized FRS could accurately predict the 10-year CVDR in NASH subjects (41). We tried to include a complete panel of CVD risk factors to empower the accuracy of our correlation analysis outputs.

The first limitation of the study was the shortage of checking for storage diseases, primary sclerosing cholangitis, and $\alpha-1$ antitrypsin, since their prevalence is too low to affect our results. The second limitation was the selection of a specific group of NAFLD patients with elevated aminotransferase levels, called as NASH. Therefore, the results could not be generalized to all NAFLD patients including those with normal aminotransferase levels, regarded as simple fatty liver. The third limitation of the 
study was the short period of follow up. Finally, we could not evaluate the effect of LFC on CVDR due to the crosssectional design of the study. Further, clinical trials regarding the response to this issue are recommended.If further research confirmed this relationship, the inclusion of LFC into the FRS formula would provide an appropriate CVDR estimation tool in NASH.

\section{Conclusion}

LFC might be correlated to CVDR in NASH patients independent of known CVD risk factors. To lower the CVDR in NASH patients, LFC reduction in accompany with other CVDR modification is recommended.

\section{Acknowledgment}

The authors would like to express their appreciation to Miss Tale and Mr. Hejrani in the research development center, Sina Hospital, for their kind assistance in preparing the draft. We also thank professors Neda Moslemi and Arsia Jamali from TUMS for a critical review of the manuscript.

\section{Conflict of Interests}

The authors declare that they have no competing interests.

\section{References}

1.Christopher D.Byrne, Giovanni Targher, NAFLD: A multisystem disease. Hepatology. 2015;62(1Supplement):S47-S64.

2. Cheung O, Sanyal AJ. Recent advances in nonalcoholic fatty liver disease. Curr Opin Gastroenterol. 2010; 26: 202-208.

3. Mariana Lazo, Jeanne M Clark. The Epidemiology of Nonalcoholic Fatty Liver Disease: A Global Perspective. Seminars in liver disease. 2008;28(4):339-350.

4. Lazo M, Hernaez R, Eberhardt MS, Bonekamp S, Kamel I, Gual-lar E, et al. Prevalence of nonalcoholic fatty liver disease in the United States: the Third National Health and Nutrition Examination Survey, 1988-1994. Am J Epidemiol. 2013;178:38-45.

5. Wong VW. Nonalcoholic fatty liver disease in Asia: a story of growth. J Gastroenterol Hepatol. 2013;28:18-23.

6. Moghaddasifar I, Lankarani KB, Moosazadeh M, Afshari M, Ghaemi A, Aliramezany M, et al. Prevalence of Non-alcoholic Fatty Liver Disease and Its Related Factors in Iran. Int J Organ Transplant Med. 2016;7(3):149-160.

7. Razavizade M, Jamali R, Arj A, Talari H. Serum parameters predict the severity of ultrasonographic findings in non-alcoholic fatty liver disease. Hepatobiliary Pancreat Dis Int. 2012;11(5):513-20.

8. Patil R, Sood GK. Non-alcoholic fatty liver disease and cardiovascular risk. World J Gastrointest Pathophysiol. 2017;8(2):51-58.

9. Soderberg C, Stal P, Askling J, Lindberg G, Marmur J, Hultcrantz R. Decreased survival of subject with elevated liver function test during a 28-year follow up. Hepatology. 2010;51:595-602.

10. Anstee QM, Targher G, Day CP. Progression of NAFLD to diabetes mellitus, cardiovascular disease or cirrhosis. Nat Rev Gastroenterol Hepatol. 2013;10:330-44.

11. Bhatia LS, Curzen NP, Calder PC, Byrne CD. Non-alcoholic fatty liver disease: a new and important cardiovascular risk factor? Eur Heart J. 2012;33:1190-200.

12. Maurantonio M, Ballestri S, Odoardi MR, Lonardo A, Loria P, Treatment of atherogenic liver based on the pathogenesis of nonalcoholic fatty liver disease: a novel approach to reduce cardiovascular risk? Arch Med Res. 2011;42:337-53.

13. Motamed N, Rabiee B, Poustchi H, Dehestani B, Hemasi GR, Khonsari MR, et al. Non-alcoholic fatty liver disease (NAFLD) and 10-year risk of cardiovascular diseases. Clin Res Hepatol Gastroenterol. 2017;41(1):31-38

14. Long MT, Wang N, Larson MG, Mitchell GF, Palmisano J, Vasan
RS, et al. Nonalcoholic fatty liver disease and vascular function: crosssectional analysis in the Framingham heart study. Arterioscler Thromb Vasc Biol. 2015;35(5):1284-91.

15. Kahl S, Straßburger K, Nowotny B, Livingstone R, Kluppelholz B, $\mathrm{Ke}$ el K, et al. Comparison of Liver Fat Indices for the Diagnosis of Hepatic Steatosis and Insulin Resistance. PLoS One. 2014;9(4):e94059.

16. Jamali R, Arj A, Razavizade M, Aarabi MH. Prediction of Nonalcoholic Fatty Liver Disease Via a Novel Panel of Serum Adipokines. Medicine (Baltimore). 2016;95(5):e2630.

17. Jamali R, Hatami N, Kosari F. The Correlation Between Serum Adipokines and Liver Cell Damage in Non-Alcoholic Fatty Liver Disease. Hepat Mon. 2016;16(5):e37412.

18. Kopin L, Lowenstein C. Dyslipidemia. Ann Intern Med. 2017;167(11):ITC81-ITC96.

19. Saklayen MG. The Global Epidemic of the Metabolic Syndrome. Curr Hypertens Rep. 2018;20(2):12.

20. Jamali R, Razavizade M, Arj A, Aarabi MH. Serum adipokines might predict liver histology findings in non-alcoholic fatty liver disease. World J Gastroenterol. 2016;22(21): 5096-103.

21. Razavizade M, Jamali R, Arj A, Matini SM, Moraveji A, Taherkhani E. The effect of pioglitazone and metformin on liver function tests, insulin resistance, and liver fat content in nonalcoholic Fatty liver disease: a randomized double blinded clinical trial. Hepat Mon. 2013;13(5):e9270.

22. Jamali R, Pourshams A, Amini S, Deyhim MR, Rezvan H, Malekzadeh R. The upper normal limit of serum alanine aminotransferase in Golestan Province, northeast Iran. Arch Iran Med. 2008;11(6):602-7.

23. Jahangiry L, Farhangi MA, Rezaei F. Framingham risk score for estimation of 10-years of cardiovascular diseases risk in patients with metabolic syndrome. J Health Popul Nutr. 2017;36(1):36.

24. Leung JY, Lin SL, Lee RS, Lam TH, Schooling CM. Framingham risk score for predicting cardiovascular disease in older adults in Hong Kong. Hong Kong Med J. 2018;24 Suppl 4(4):8-11.

25. Khalili D, Hadaegh F, Soori H, Steyerberg EW, Bozorgmanesh M, Azizi F. Clinical Usefulness of the Framingham Cardiovascular Risk Profile Beyond Its Statistical Performance: The Tehran Lipid and Glucose Study. Am J Epidemiol. 2012;176(3):177-186.

26. Cheung CL, Lam KS, Wong IC, Cheung MB. Non-invasive score identifies ultrasonography diagnosed non-alcoholic fatty liver disease and predicts mortality in the USA. BMC Med. 2014;12(154).

27. Cho EJ, Han K, Lee SP, Shin DW, Yu SJ. Liver enzyme variability and risk of heart disease and mortality: A nationwide population-based study. Liver Int. 2020 Mar 9. doi: 10.1111/liv.14432. [Epub ahead of print]

28. Wattacheril J. Extrahepatic Manifestations of Nonalcoholic Fatty Liver Disease. Gastroenterol Clin North Am. 2020;49(1):141-149.

29. Brunner KT, Pedley A, Massaro JM, Hoffmann U, Benjamin EJ, Long MT. Increasing Liver Fat Is Associated With Incident Cardiovascular Risk Factors. Clin Gastroenterol Hepatol. 2019 Aug 9. pii: S1542-3565(19)30859-6.

30. Stahl EP, Dhindsa DS, Lee SK, Sandesara PB, Chalasani NP, Sperling LS. Nonalcoholic Fatty Liver Disease and the Heart: JACC State-of-the-Art Review. J Am Coll Cardiol. 2019;73(8):948-963.

31. Byrne CD, Targher G. NAFLD: a multisystem disease. J Hepatol. 2015;62(1 Suppl):S47-64

32. Dogan S, Celikbilek M, Yilmaz YK, Sarikaya S, Zararsiz G, SerinHI, et al. Association between liver fibrosis and coronary heart disease risk in patients with nonalcoholic fatty liver disease. Eur J Gastroenterol Hepatol. 2015;27: 298-304.

33. McKimmie RL, Daniel KR, Carr JJ, Bowden DW, Freedman BI, Register TC, Hsu FC, et al. Hepatic steatosis and subclinical cardiovascular disease in a cohort enriched for type 2 diabetes: The Diabetes Heart Study. Am J Gastroenterol. 2008;103:3029-3035.

34. Lauridsen BK, Stender S, Kristensen TS, Kofoed KF, Køber L, Nordestgaard BG, et al. Liver fat content, non-alcoholic fatty liver disease, and ischemic heart disease: Mendelian randomization and meta-analysis of 279013 individuals. Eur Heart J. 2018;39(5):385393.

35. Pacifico L, Anania C, Martino F, Cantisani V, Pascone R, Marcantonio A, et al. Functional and morphological vascular changes in pediatric nonalcoholic fatty liver disease. Hepatology. 2010;52:164351.

36. Sayki Arslan M, Turhan S, Dincer I, Mizrak D, Corapcioglu D, 
Idilman R. A potential link between endothelial function, cardiovascular risk, and metabolic syndrome in patients with Nonalcoholic fatty liver disease. Diabetol Metab Syndr. 2014;6:109.

37. Byrne CD, Targher G. NAFLD: a multisystem disease. J Hepatol. 2015;62:S47-64.

38. Oni ET, Agatston AS, Blaha MJ, Fialkow J, Cury R, Sposito A, et al. A systematic review: burden and severity of subclinical cardiovascular disease among those with nonalcoholic fatty liver; should we care? Atherosclerosis. 2013;230:258-67.

39. Kabisch S, Bäther S, Dambeck U, Kemper M, Gerbracht C, Honsek C, et al. Liver Fat Scores Moderately Reflect Interventional Changes in Liver Fat Content by a Low-Fat Diet but Not by a Low-Carb Diet. Nutrients. 2018;10(157).

40. Centis E, Marzocchi R, Domizio SD, Ciaravella MF, Marchesini G. the Effect of Lifestyle Changes in Non-Alcoholic Fatty Liver Disease. Dig Dis. 2010;28:267-273.

41. Treeprasertsuk S, Leverage S, Adams LA, Lindor KD, St SauverJ, Angulo P. The Framingham risk score and heart disease in nonalcoholic fatty liver disease. Liver Int. 2012;32:945-50. 